\title{
Spectra of Hydrogen Atom with GUP and Extra Dimensions
}

\author{
Benrong Mu \\ School of Physical Electronics, University of Electronic Science and Technology of China, Chengdu, Sichuan, China \\ Email: mubenrong@uestc.edu
}

Received 2013

\begin{abstract}
We argue that the Generalized Uncertainty Ptinciple (GUP) and the compact Extra dimensions will lead the hydrogen atom has the non bounded state spectra which equal the free particle in extra dimensions. We use Generalized Uncertainty Principle (GUP) to calculate the new energy spectra of hydrogen atom, and which lives in three dimensional Euclidean spaces with one extra dimension. The result is not familiar with our known before that $E_{n}=0$ when $n$ is large enough. In our modified new spectra, we obtain that $E_{n, \ell, 0}=\left(1+2 \beta \ell^{2} / \rho^{2}\right) \ell^{2} / 2 m \rho^{2}$, which is lager than zero. These new spectra give us new method to obtain the existence of extra dimensions. Finally, we find that the spectra are same as part of extra dimensions in planck scale.
\end{abstract}

Keywords: Hydrogen Atom; GUP; Extra Dimensions

\section{Introduction}

There exist many theories for solving the problem in unifying the quantum theory and gravity, such as string theory and loop quantum theory, which all of them predict that there exits the minimal observable length which proportional to the Planck length $\ell_{p} \sim 10^{-33} \mathrm{~cm}$. [1] This minimal length was emerged by our observation method under high energy scale, and which phenomenon is not surprised for us. In usual quantum mechanics, which does not consider the effect of gravity, has the Heisenberg Uncertainty Principle which is described as $\Delta x \Delta p \geq 1 / 2$. This uncertainty principle implies that the large $\Delta p$ could make the position distance $\Delta x$ arbitrary small. However, when we consider the gravity, which becomes more important in Planck scale, cannot lead us to neglect the effect of gravity in many special cases. This motivation gives us the advices that when we use high energy to observe any arbitrary small scale, it will causes the minimal blank hole, and hence its horizon becomes the minimal observable length naturally. In this analysis, the Heisenberg Uncertainty Principle must be modifed for including the effects of quantum gravity and the one of the most important models of this case is the Generalized Uncertainty Principle (GUP). The modified fundamental commutator $(\hbar=c=1)$ is [2-5]

$$
[x, p]=\text { if }(p)
$$

This new commutator relation gives us the possibilities to contain the different conditions, which could describe the usual uncertainty principle in our familiar quantum mechanics that $f(p)=1$ and also is will includes the nonzero position distance in high energy scale. In our requirements, $f(p)$ is a positive function and which can be expanded by Taylor series as $f(p)=1+\beta p^{2}+o\left(p^{4}\right)$, and $\beta=\beta_{0} \ell_{p}^{2}=\beta_{0} / M_{p}^{2}$. Our previous work had been introduced the running constant $\beta_{0}$ of $f(p)$ that would varied with energy scales and be combined with another quantum gravity quantity: extra dimensions [6]. These characteristics make us to construct the low energy effective theory which will contains some quantum gravity phenomenon in intermediate energy scale. In GUP model, we choose the first two terms as

$$
[x, p]=i\left(1+\beta p^{2}\right)
$$

and hence, the GUP model is

$$
\Delta x \Delta p \geq i\left[1+\beta(\Delta p)^{2}\right]
$$

Generalizing the commutator to higher dimensions and expressing it as tensor formalism, we obtain that

$$
\left[x_{i}, p_{j}\right]=i\left(\delta_{i j}+\beta \delta_{i j} p^{2}+2 \beta p_{i} p_{j}\right)
$$

and the correspondent higher dimensional GUP model is

$$
\Delta x_{i} \Delta p_{i} \geq \frac{1}{2}\left[1+\beta\left((\Delta p)^{2}+\langle p\rangle^{2}\right)+2 \beta\left(\Delta p_{i}^{2}+\left\langle p_{i}\right\rangle^{2}\right)\right]
$$


where $p^{2}=\sum p_{i} p_{i}$. This uncertainty principle easily deduces the minimal observable length that $\Delta\left(x_{i}\right)_{\min } \sim$ $\sqrt{\beta_{0}} \ell_{p}$ for every direction.

In this paper, we construct the toy model which the extra dimension is only considered without any other potentials, and use the GUP to redescribe the hydrogen atom. This assumption is not unjustifiable in view of the fact that the standard model charges cannot move in extra dimensions in theory of brane world model. Now, extra dimensions are widely accepted by theorists and there are large works about it [7-13]. This model gives us the new spectra, which looks same as our previous works [6], however, have different physical meaning. In usual spectra of hydrogen atom, we know the spectra is given as

$$
E_{n}=-\left[\frac{m}{2}\left(\frac{e^{2}}{4 \pi \varepsilon_{0}}\right)\right] \frac{1}{n^{2}}
$$

We know that the ground state energy is $E_{1}=-13.6 \mathrm{eV}$. When $n \rightarrow \infty$, we obtain the upper bound of the energy is zero. In our following works, when consider the term of one extra dimension $\ell^{2} / 2 m \rho^{2}$, the upper bound of hydrogen atom will be larger than zero. On the other hand, we obtain the energy spectra of hydrogen atom is same as the spectra which describe the particle move in the extra dimension freely in Planck scale. To sum up, it gives us the new detect method to find the existence of extra dimensions in intermediate energy scale.

\section{Method}

In our case, we need that the wave function obeys the periodical condition $\psi(x, y)=\psi(x, y+2 \pi \rho)$, where $x$ represents the familiar Euclidean space and $y$ yrepresents the compact space. As we known, the function can be described as the production of two parts that $\psi(x, \theta)=$ $\phi(x) \Theta(\theta)$, where $\Theta$ represents the wave function in compact space, and we have $\Theta(\theta)=\Theta(\theta+2 \pi)$. In this way, the familiar wave function can be divided into two parts. We hence can calculate the energy spectrum of Euclidean and compact space respectively, and count up these two parts for obtaining the total energy of the system.

$$
\begin{aligned}
& x_{i}=x_{0 i} \\
& p_{i}=p_{0 i}\left(1+\beta p_{0}^{2}\right), p_{0 i}=-i \frac{d}{d x_{0 i}}
\end{aligned}
$$

where $p_{0}^{2}=\sum p_{0 i} p_{0 i}$ and $\left[x_{0 i}, p_{0 j}\right]=i \hbar \delta_{i j}$, the usual canonical operators. The unperturbed schrödinger equation reads

$$
\left[\frac{p^{2}}{2 m}+V(\vec{x})\right] \psi=E \psi
$$

with modifying the commutator relations, we can obtain the new Hamiltonian of this quantum system that

$$
H=H_{0}+H_{1}=\frac{p_{0}^{2}}{2 m}+V(\vec{x})+\frac{\beta}{m} p_{0}^{4}
$$

the first order correction is then given as

$$
\begin{aligned}
E_{n}^{(1)} & =\frac{\beta}{m}\left\langle\psi\left|p_{0}^{4}\right| \psi\right\rangle=4 m \beta\left\langle\left(E_{n}^{(0)}-V\right)^{2}\right\rangle \\
& =4 m \beta\left[\left(E_{n}^{(0)}\right)^{2}-2 E_{n}^{(0)}\langle V\rangle+\left\langle V^{2}\right\rangle\right]
\end{aligned}
$$

\section{Modified Spectra of Hydrogen Atom}

The schrödinger equation of hydrogen atom is

$$
\frac{p^{2}}{2 m} \psi+V(\vec{r}) \psi=E \psi
$$

where potential $V(\vec{r})$ comes from the Coulomb's law and be given as

$$
V(\vec{r})=-\frac{e^{2}}{4 \pi \varepsilon_{0}} \frac{1}{r}
$$

As we mentioned in introduction, we should consider the hydrogen atom with an extra dimension at first. When we consider the extra dimension, the term $p^{2}$ of schrödinger equation should contain the momentum in extra dimension and which can be rewritten as

$$
p^{2}=\nabla^{2}+\frac{1}{\rho^{2}} \frac{d^{2}}{d \theta^{2}}
$$

where the correspondent wave function is $\psi=\Theta(\theta) \phi(r, \varphi, \omega)$. In this way, the equation be divided into two equations which are unperturbed as follow

$$
\begin{aligned}
& -\frac{1}{2 m \rho^{2}} \frac{d^{2} \Theta}{d \theta^{2}}=E_{\ell}^{(0)} \\
& -\frac{1}{2 m} \nabla^{2} \phi+V(\vec{r}) \phi=E_{n}^{(0)} \phi
\end{aligned}
$$

so, we have the unperturbed energy spectra of hydrogen atom with one extra dimension, which can be given as

$$
\begin{aligned}
E_{n, \ell}^{(0)} & =E_{n}^{(0)}+E_{\ell}^{(0)} \\
& =-\left[\frac{m}{2}\left(\frac{e^{2}}{4 \pi \varepsilon_{0}}\right)^{2}\right] \frac{1}{n^{2}}+\frac{\ell^{2}}{2 m \rho^{2}}
\end{aligned}
$$

Now, let us modify the commutation relations by using GUP, we obtain the perturbed term of Hamiltonian that $H_{1}=\beta p_{0}^{4} / \mathrm{m}$. So, we find the first order correction of energy spectra is

$$
\begin{aligned}
E_{n, \ell, s}^{(0)} & =4 m \beta\left[\left(E_{n, \ell}^{(0)}\right)^{2}-2 E_{n, \ell}^{(0)}\langle V\rangle+\left\langle V^{2}\right\rangle\right] \\
& =4 m \beta\left[\left(E_{n, \ell}^{(0)}\right)^{2}-2 E_{n, \ell}^{(0)}\left(\frac{e^{2}}{4 \pi \varepsilon_{0}}\right)\left\langle\frac{1}{r}\right\rangle+\left(\frac{e^{2}}{4 \pi \varepsilon_{0}}\right)^{2}\left\langle\frac{1}{r^{2}}\right\rangle\right]
\end{aligned}
$$


where

$$
\left\langle\frac{1}{r}\right\rangle=\frac{1}{n^{2} a} \text { and }\left\langle\frac{1}{r^{2}}\right\rangle=\frac{1}{(s+1 / 2) n^{3} a^{2}}
$$

Finally, we obtain the modified energy spectra of hydrogen atom that

$$
\begin{aligned}
E_{n, \ell, s}= & E_{n, \ell}^{(0)}+4 m \beta\left[+\left(\frac{e^{2}}{4 \pi \varepsilon_{0}}\right)^{2} \frac{1}{(s+1 / 2) n^{3} a^{2}}\right] \\
= & E_{n}^{(0)}\left[1+4 m \beta\left(\frac{2 b}{n^{2} a}+E_{n}^{(0)}\right)\right] \\
& +\left[\begin{array}{l}
1+8 m \beta\left(\frac{b}{n^{2} a}+E_{n}^{(0)}\right) \\
n^{2} a
\end{array}\right] \\
\left.+2 \beta \frac{\ell^{2}}{\rho^{2}}\right] \frac{\ell^{2}}{2 m \rho^{2}} & {\left[\frac{b^{2}}{\left(s+\frac{1}{2}\right) n^{3} a^{2}}\right] }
\end{aligned}
$$

When $n$ is large enough, in our introduction before, will gives us $E_{n} \rightarrow 0$. But, we obtain new value in here when $n$ is large, the spectra is

$$
E_{n, \ell, 0}=\left(1+2 \beta \frac{\ell^{2}}{\rho^{2}}\right) \frac{\ell^{2}}{2 m \rho^{2}}
$$

This result is too surprised. As we known, the usual ground state of hydrogen atom is $-13.6 \mathrm{eV}$. With energy level increase, the value of energy go up to, however, specially, there exits the upper bound when $n$ is large enough which equals zero. But, our result introduction the new spectra of hydrogen atom when $n$ is large. Above the upper bound, we find new spectra! This result is not end of journey, noteworthily, as our works before, we have the relations $\sqrt{\beta} \sim \rho$ in Planck scale. It express that

$$
E_{n, \ell, 0}=\left(1+2 \ell^{2}\right) \frac{\ell^{2}}{2 m \rho^{2}}
$$

which is the energy spectra of extra dimension. Considering the physical meaning of the nonzero position distance in Planck scale, there exists the minimal black hole and the horizon is that nonzero point. This condition lead the hydrogen atom has unbounded state in extra dimensions and which is reason that we obtain the positive spectra in final.

\section{Summary}

We use GUP and extra dimensions models to rewritten the equation of hydrogen atom and obtain the modified energy spectra which is far from the familiar solution that has a upper bound. In this modified spectra, we find that there also exits discrete spectra in field of $E>0$, which is same as the free particle that lives in extra dimensions. This result is not familiar in usual hydrogen atom. As this term is contributed by the wave function in extra dimension, it introduces the new method to find extra dimensions in intermediate energy scale.

\section{REFERENCES}

[1] G. Veneziano, “A Stringy Needs Just Two Constants," Europhysics Letters, Vol. 2, No.3, 1986, p. 199. doi:10.1209/0295-5075/2/3/006

[2] D. J. Gross and P. F. Mende, "String Theory The Planck Scale,” Nuclear Physics B, Vol. 303, No. 3, 1988, pp. 407-454.doi:10.1016/0550-3213(88)90390-2

[3] D. Amati, M. Ciafaloni and G. Veneziano, "Can Spacetime Be Probed Below the String Size,” Physics Letters, B, Vol. 216, No. 1-2, 1989, pp. 41-47. doi:10.1016/0370-2693(89)91366-X;

[4] K. Konishi, G. Pa_uti and P. Provero, "Minimum Physical Length and the Generalized Uncertainty Principle in String Theory,” Physics Letters B, Vol. 234, No. 3, 1990, pp. 276-284. doi:10.1016/0370-2693(90)91927-4

[5] R. Guida, K. Konishi and P. Provero, "On the Short Distance Behavior of String Theories," Modern Physics Letters A, Vol. 06, No. 16, 1991, p. 1487. doi:10.1142/S0217732391001603

[6] M. Maggiore,“ A Generalized Uncertainty Principle in Quantum Gravity,” Physics Letters B, Vol. 304, No. 1-2, 1993, pp. 65-69. doi:10.1016/0370-2693(93)91401-8

[7] A. Kempf, G. Mangano and R. B. Mann, "Hilbert Space Representation of the Minimal Length Uncertainty Relation,” Physical Review D, Vol. 52, 1995, pp. 1108-1118. doi:10.1103/PhysRevD.52.1108

[8] A. Kempf, "Non-Pointlike Particles in Harmonic Oscillators," Journal of Physics A:Mathematical and General, Vol. 30, No. 6, 1997, p. 2093.

doi:10.1088/0305-4470/30/6/030

[9] F. Brau, "Minimal Length Uncertainty Relation and the Hydrogen Atom," Journal of Physics A: Mathematical and General, Vol. 32, No. 44, 1999. doi:10.1088/0305-4470/32/44/308

[10] F. Brau and F. Buisseret, "Minimal Length Uncertainty Relation and Gravitational Quantum Well,” Physical Review $D$, Vol. 74, 2006, 036002. doi:10.1103/PhysRevD.74.036002

[11] L. N. Chang, D. Minic, N. Okamura and T. Takeuchi, "Exact Solution of the Harmonic Oscillator in Arbitrary Dimensions with Minimal Length Uncertainty Relations," Physical Review D, Vol. 65, 2002, 125027. doi:10.1103/PhysRevD.65.125027

[12] I. Dadic, L. Jonke and S. Meljanac, "Harmonic Oscillator With Minimal Length Uncertainty Relations and Ladder Operators,” Physical Review D, Vol. 67, 2003, 087701 doi:10.1103/PhysRevD.67.087701

[13] K. Nozari and T. Azizi, "Some Aspects of Gravitational Quantum Mechanics,” General Relativity and Gravitation, Vol. 38, No. 38, 2006, pp. 735-742. 
doi:10.1007/s10714-006-0262-9

[14] M. M. Stetsko, "Corrections to the ns Levels of The Hydrogen Atom in Deformed Space With Minimal Length," Physical Review A, Vol. 74, No. 6, 2006, 062105. doi:10.1103/PhysRevA.74.062105

[15] S. Z. Benczik, "Investigations on the Minimal-Length Uncertainty Relation,” Dissertation for Doctor of Philosophy.

[16] M. V. Battisti and G. Montani, “The Big-Bang Singularity in the Framework of a Generalized Uncertainty Principle,” Physics Letters B, Vol. 656, No. 1-3, 2007, pp. 96-101.doi:10.1016/j.physletb.2007.09.012.

[17] M. V. Battisti and G. Montani, Physics Review D, Vol. 77, 2008,023518.

[18] M. V. Battisti, arXiv: 0805.1178.

[19] S. Das and E. C. Vagenas, Physics Review Letters, Vol. 101, 2008, 221301.

[20] B. Mu, H. Wu and H. Yang, arXiv:0909.3635 [hep-th].

[21] B. Mu, "Spectra of harmonic oscillators with GUP and extra dimensions," to appear.

[22] I. Antoniadis, "A Possible New Dimension at a Few TeV," Physics Letters B, Vol. 246, No. 3-4, 1990, pp. 377-384. doi:10.1016/0370-2693(90)90617-F

[23] N. Arkani-Hamed, S. Dimopoulos and G. R. Dvali, “The Hierarchy Problem and New Dimensions at a Millimeter," Physics Letters B, Vol. 429, No. 34, 1998, pp. 263-272.doi:10.1016/S0370-2693(98)00466-3
[24] I. Antoniadis, N. Arkani-Hamed, S. Dimopoulos and G. R. Dvali, "New Dimensions at a Millimeter to a Fermi and Superstrings at a Tev," Physics Letters B, Vol. 436, No. 3-4,1998, pp. 257-263. doi:10.1016/S0370-2693(98)00860-0

[25] L. Randall and R. Sundrum, "Large Mass Hierarchy from A Small Extra Dimension,” Physical Review Letters, Vol. 83, 1999, pp. 3370-3373. doi:10.1103/PhysRevLett.83.3370

[26] L. Randall and R. Sundrum, "An Alternative to Compactification,” Physical Review Letters, Vol. 83, 1999, pp.4690-444693. doi:10.1103/PhysRevLett.83.4690

[27] G. R. Dvali, G. Gabadadze and M. Porrati, “4D Gravity On A Brane in 5D Minkowski Space,” Physics Letters B, Vol. 485, No. 1-3, 2000, pp. 208-214. doi:10.1016/S0370-2693(00)00669-9

[28] T. Appelquist, H. C. Cheng and B. A. Dobrescu, "Bounds on Universal Extra Dimensions," Physical Review D, Vol. 64, 2001, 035002. doi:10.1103/PhysRevD.64.035002

[29] D. Cremades, L. E. Ibanez and F. Marchesano, "Standard Model at Intersecting D5-Branes:Lowering the String Scale," Nuclear Physics B, Vol. 643, No. 1-3, 2002, pp. 93-130. doi:10.1016/S0550-3213(02)00746-0

[30] C. Kokorelis, "Exact Standard Model Structures From Intersecting D5-Branes,” Nuclear Physics B, Vol. 677, No. 1-2, 2004, pp. 115-163. doi:10.1016/j.nuclphysb.2003.11.007 